\title{
Analyse phytosociologique de la végétation de la réserve naturelle forestière de Vyanda au Sud-Ouest du Burundi
}

\author{
André NDUWIMANA ${ }^{1 *}$, Richard HABONAYO ${ }^{1}$, Blaise NDAYIZEYE ${ }^{2}$ et \\ Mathias HITIMANA ${ }^{2}$ \\ ${ }^{1}$ Université du Burundi, Faculté d'Agronomie et de Bio-Ingénierie, Centre de Recherche en Sciences des \\ Productions Animales, Végétales et Environnementales (CRAVE), B.P 2940 Bujumbura, Burundi. \\ ${ }^{2}$ Université du Burundi, Faculté des Sciences, Département de Biologie, BP 2700 Bujumbura, Burundi. \\ *Auteur pour les correspondances ; E-mail: andnduwi@yahoo.fr ; andre.nduwimanal@ub.edu.bi ; \\ Tél: +257 79947367.
}

\begin{tabular}{ccc}
\hline Received: 24-05-2021 & Accepted: 22-07-2021 & Published: 31-08-2021 \\
\hline
\end{tabular}

\section{RESUME}

La réserve naturelle forestière de Vyanda subit des pressions anthropiques permanentes et risque de disparaître avant qu'elle ne soit étudiée. La présente étude a pour objectif de déterminer la composition et la diversité de la végétation de cette réserve et de motiver la prise des mesures de conservation localement adaptées. L'étude a procédé par l'inventaire de la végétation suivant la méthode du relevé phytosociologique de Braun Blanquet (1932). Des spectres bruts et pondérés des formes biologiques, des types de diaspores et des types phytogéographiques ont été établis. Les résultats de l'inventaire floristique ont fait état de 108 espèces réparties en 81 genres et 41 familles. Sur le plan des formes biologiques et des types de diaspores, les phanérophytes et les sarcochores dominent tandis que les hémicryptophytes de même que les sclérochores sont bien représentés. Sur le plan phytogéographique, l'étude a mis en évidence une forte représentativité de l'élément paléo-africain, de l'élément soudano-zambézien et de l'élément guinéo-congolais. Ces spectres attestent la spécificité de cette réserve comme forêt claire du type miombo tout en mettant en évidence l'impact des perturbations dont elle est victime. L'étude met en évidence la richesse spécifique de la réserve et démontre l'intérêt qu'il y a de prendre des mesures visant à arrêter les perturbations anthropiques et à bien gérer ce milieu naturel. Sa diversité est en effet grande comparé aux autres réserves naturelles du voisinage.

(C) 2021 International Formulae Group. All rights reserved.

Mots clés : Végétation, Vyanda, Burundi, forêt claire, spectre pondéré.

\section{Phytosociological analysis of the vegetation of the Vyanda natural forest reserve in southwestern Burundi}

\begin{abstract}
The Vyanda natural forest reserve is under constant human pressure and is in danger of disappearing before it is studied. The objective of this study is to determine the composition and diversity of the vegetation of this reserve and to motivate the taking of locally adapted conservation measures. The study carried out a vegetation inventory using the Braun Blanquet (1932) phytosociological approach. Spectra of life forms,
\end{abstract}


phytogeographical elements and diaspores morphology were established. The results of the floristic inventory revealed 108 species divided into 81 genera and 41 families. In terms of life forms and types of diaspores, phanerophytes and sarcochores dominate when hemicryptophytes as well as sclerochores are well represented. Phytogeographically, the study revealed a strong representativeness of the Paleo-African element, the SudanoZambezian element and the Guinean-Congolese element. These spectra attest to the specificity of Vyanda natural forest reserve as an open forest of the miombo type while highlighting the impact of the disturbances that affect it. The study highlights the specific richness of the reserve and its diversity and demonstrates an urgent need to take measures to stop anthropogenic disturbances and well managing this natural ecosystem. Its diversity is indeed great compared to other natural reserves in the region

(C) 2021 International Formulae Group. All rights reserved.

Keywords: Vegetation, Vyanda, Burundi, open forest, weighted spectrum.

\section{INTRODUCTION}

Le Burundi est un pays d'Afrique centrale dont le relief varié a permis l'existence d'écosystèmes diversifiés comprenant des forêts ombrophiles de montagne, des galeries forestières, des forêts claires, des savanes et des pelouses (Habonimana et al., 2011). Ces milieux naturels sont confrontés à d'importantes menaces occasionnées surtout par une croissance accélérée de la population qui provoque une perte de la biodiversité et une modification des habitats en termes de composition et configuration (Ndayishimiye et al, 2018).

La stratégie adoptée par le Burundi pour stopper cette érosion de la biodiversité fut la création d'un grand nombre d'aires protégées, la conservation de la biodiversité étant difficile à appliquer en dehors des aires protégées à cause des activités humaines (Gueulou et al., 2019). Ainsi, malgré sa petite superficie de $27834 \mathrm{~km}^{2}$, le Burundi en arrive à 17 aires protégées (Masharabu, 2011) dont la réserve forestière de Vyanda (objet de la présente étude) qui formait, avec la réserve de Nkayamba et celle de Nyamirambo, la réserve forestière de Rumonge au Sud-Ouest du Burundi (Habonimana et al., 2008). Cette dernière a été décrite comme une formation de forêt claire du type miombo dominée par les genres Brachystegia et Uapaca (Nzigidahera, 2000) et les études détaillées (Habonimana et al, 2010) n'ont pas touché la partie réserve naturelle forestière de Vyanda probablement par biais d'échantillonnage parce que cette dernière n'est pas proche de la route.
La réserve naturelle de Vyanda a été créée dans un contexte conflictuel (INECN, 2008) et subit des pressions anthropiques permanentes caractérisées entre autres par le défrichement cultural, les feux de brousse et les coupes abusives du bois. Une étude scientifique est plus qu'urgente pour connaitre l'état de sa biodiversité et fournir des éléments de décision pour sa gestion durable. L'insuffisance des données scientifiques est en effet une contrainte majeure qui limite la mise en œuvre des mesures d'aménagement et de gestion durable de la biodiversité (Abdourhamane et al., 2013). C'est dans ce contexte que cette recherche a été entreprise. Elle se fixe comme objectif principal de mettre à la disposition des gestionnaires et décideurs des données fiables pour éclairer les choix en matière de conservation.

\section{MATERIEL ET METHODES Milieu d'étude}

$\mathrm{La}$ réserve naturelle forestière de Vyanda (RNFV) est située dans la partie méridionale du Burundi (entre $4^{\circ}$ et $4^{\circ} 10^{\prime}$ de latitude Sud ; $29^{\circ} 32^{\prime}$ et $29^{\circ} 37^{\prime}$ de longitude Est), à cheval entre les communes de Vyanda et de Rumonge (Figure 1). Sa superficie à la création est estimée à 4500 ha dont 2000 ha pour la forêt naturelle et 2500 ha pour les plantations de Pinus.

L'étude s'est déroulée dans la partie naturelle de cette réserve qui se répartit sur deux régions naturelles dénommées l'Imbo et le Mumirwa. La zone d'étude connait un climat du type AW4s selon la classification de Köppen caractérisé par huit mois de saison des 
pluies et quatre mois de saison sèche. $\mathrm{La}$ température moyenne est de $24{ }^{\circ} \mathrm{C}$, la pluviosité moyenne est de $1050 \mathrm{~mm}$ pour la région de l'Imbo contre $1170 \mathrm{~mm}$ pour la région du Mumirwa (OBPE, 2019 ; Hakizimana et al., 2011).

La zone d'étude se localise entre les altitudes de $800 \mathrm{~m}$ à $1400 \mathrm{~m}$ et a été choisie pour son caractère naturel et par le fait qu'elle est accessible via la Route Nationale numéro 3: Bujumbura- Kigwena $(96 \mathrm{~km})$. Elle est presque partout entourée de villages (Kigwena, Cabara, Karonda, Mugara, Kigutu) dont la population renforcée par l'installation des rapatriés y exerce des activités perturbatrices.

\section{Collecte des données}

La collecte des données de la végétation a été faite par la technique de relevés suivant la méthode phytosociologique de BraunBlanquet (1932). En se fondant sur des inventaires floristiques, la phytosociologie permet de décrire précisément une communauté végétale en fonction de sa composition spécifique, reflet des caractéristiques du milieu (Gérard, 2012; Dengler et al, 2016). Elle est choisie comme méthode de terrain car elle assure la cohérence au sein des bases de données d'inventaires floristiques.

Les données ont été collectées dans 34 placettes carrées de $20 \mathrm{~m}$ de côté (Idjigberou et Tychon, 2017) installées le long des transects établis sur les collines jugées les plus représentatives de la végétation sur base de la physionomie. Ces placettes étaient centrées sur les différents transects et celles se trouvant sur un même transect étaient équidistantes de 250 m. Au total sept transects totalisant une longueur de $8.132 \mathrm{~m}$ ont été installés. Parmi ceux-ci, six ont été orientés dans la direction Ouest-Est et le dernier dans la direction SudNord au moyen d'une boussole de marque Suunto. Ces transects ont été installés chaque fois à $50 \mathrm{~m}$ de la bordure de la forêt pour éviter l'effet de bordure dans les données collectées.

Au niveau de chaque station, deux types d'informations ont été notées :

- Les observations grossières des conditions écologiques hiérarchisées suivant 5 niveaux pour l'altitude (altitude entre 950 et $1000 \mathrm{~m}$, entre 1000 et $1050 \mathrm{~m}$, entre 1050 et $1100 \mathrm{~m}, 1100$ et $1150 \mathrm{~m}$ et altitude au-delà de $1150 \mathrm{~m}$ ), 4 niveaux pour la structure du sol (sol à limon, sol à limon et sable, à sol à limon et cailloux, sol sableux), 3 niveaux pour la pente (pente modérée, pente forte, pente abrupte) et trois niveaux pour ce qui concerne la localisation (bas, milieu, sommet) sur la colline. Toute la zone présente des signes d'une perturbation anthropique par le feu et par coupe, raison pour laquelle cette variable n'a pas été utilisée car apparemment constante

- Celles concernant la végétation et visant le recouvrement, la liste des espèces et les coefficients d'abondance-dominance suivant l'échelle de Braun-Blanquet (1932).

Sur le terrain, des spécimens de toutes les espèces recensées ont été récoltés et mis en herbier. La détermination des noms scientifiques des espèces a été faite grâce à l'Herbarium de l'Université du Burundi (BJA) et à l'ouvrage de Reekmans et Niyongere (1983). Les noms scientifiques des espèces ainsi que ceux des familles ont été validés après la vérification des noms actualisés dans la base de données des plantes africaines sur le site web http://www.ville-ge.ch/musinfo/bd/cjb/ africa/recherche.php. La collecte des données a eu lieu en 2019 (de janvier à avril).

\section{Traitement et analyse des données}

L'opération de traitement des données phytosociologiques a consisté en la transformation des coefficients d'abondancedominance des espèces en une valeur semiquantitative Ri de recouvrement (Gillet, 2000) qui exprime la dominance de chaque espèce dans son milieu.

$$
R i=\frac{\sum_{i=1}^{n} R m i}{N}
$$

Où : Ri = Valeur semi-quantitative de l'espèce $\mathrm{i} ; \mathrm{Rmi}=$ «Recouvrement moyen $»$ de l'espèce i dans un relevé (obtenu à partir de la conversion du code de Braun-Blanquet) ; $\mathrm{n}=$ nombre de relevés où l'espèce $\mathrm{i}$ est présente ; $\mathrm{N}=$ Nombre de tous les relevés.

L'analyse de la flore a d'abord porté sur l'établissement de la composition du milieu en espèces (Richesse spécifique) et dans le calcul 
des indices de diversités: Indice $\left(\mathrm{H}^{\prime}\right)$ de diversité de Shannon-Weaver (1949), de Simpson (D) et de régularité (équitabilité) de Piélou (E).

$$
\begin{aligned}
& H^{\prime}=-\sum_{i=1}^{s} P_{i} \cdot \log _{2} P_{i} \\
& D=\sum_{i=1}^{s}\left(P_{i}\right)^{2} \\
& E=\frac{H^{\prime}}{\log _{2} S}
\end{aligned}
$$

Avec $s$ égal au nombre total des espèces; $\mathrm{P}_{\mathrm{i}}$ est l'abondance proportionnelle de chaque espèce et $\mathrm{N}$ le nombre total d'individus. Pour les données phytosociologiques, $P_{i}=\frac{R_{i}}{\sum_{i=1}^{S} R_{i}} \quad$ et $N=\sum_{i=1}^{s} R_{i}$

L'utilisation de ces trois indices de manière concomitante permet une étude plus complète des informations concernant la structure des communautés (Grall et Coïc, 2005).

L'analyse de la flore a également fait recours à des descripteurs non taxonomiques (Types biologiques, types de diaspores, types phytogéographiques).

Pour les types biologiques (TB), nous avons utilisé le système de Raunkiær (1934) modifié par Lebrun (1947) qui reconnaît les phanérophytes $(\mathrm{Ph})$, les chaméphytes $(\mathrm{Ch})$, les hémichryptophytes (Hc), les Thérophytes (Th), les Géophytes (Gé) et les Hydrophytes (Hy).

Pour les types phytogéographiques, nous avons utilisé le système de Lebrun (1947) modifié (White, 1979 ; White, 1983) où on reconnaît les espèces à large distribution sur le globe terrestre (Cos), espèces pantropicales $(\mathrm{Pan})$; espèces paléo-tropicales $(\mathrm{Pal})$; espèces afrotropicales (Afr trop); espèces plurirégionales africaines (Plur Afr); espèces montagnardes (Mo), les epèces guinéocongolaises (GC), les espèces de liaisons ( $\mathrm{Li}$ Mo-SZ, Li SZ-GC); espèces soudano zambéziennes (SZ).

Pour les types de diaspores, nous avons utilisé le système de classification de Dansereau et Lems (1957) qui reconnaît les sarcochores (sarco), les desmochores (Desmo), les sclérochores (Scléro), les ptérochores (Ptéro), les pogonochores (Pogo), les ballochores (Ballo), les barochores (Baro) et les hydrochores (Hy).

Pour ces descripteurs non taxonomiques, il a été établi les spectres bruts et pondérés.

L'Analyse de la flore a ensuite procédé par l'identification des groupements et leur caractérisation.

L'identification des groupements a été faite à l'aide de la classification ascendante hiérarchique (CAH) par la méthode de Ward d'un tableau de 34 relevés et 48 espèces sur base du critère de présence /absence des espèces.

La méthode de Ward a été choisie car elle est jugée la plus puissante parmi les méthodes d'agrégation et la plus utilisée (Morey et al., 1983).

Nous avons utilisé le tableau espèces / relevés duquel on a provisoirement retiré celles qui ne sont présentes que dans 2 relevés pour éliminer le bruit que causeraient ces espèces rares à la classification (Gillet, 2000 ; Meddour, 2011).

On a également procédé à l'ordination par la méthode d'analyse factorielle des correspondances (AFC). Nous l'avons choisie parce qu'elle est jugée méthode la plus appropriée (Meddour, 2011) quand on travaille sur des données qualitatives (Roux, 2006).

La comparaison des groupements identifiés a été réalisée par l'établissement de l'indice de similitude de Sorensen :

$S_{8}=\frac{2 a}{2 a+b+c}$

Avec $\mathrm{a}:$ le nombre d'espèces communes, $\mathrm{b}$ et $\mathrm{c}:$ le nombre d'espèces appartenant seulement à un groupement et l'autre groupement comparés.

La confirmation du rôle des facteurs écologiques dans la répartition des espèces est réalisée par le test de permutation de l'analyse canonique des correspondances (ACC) réalisée avec les observations sur les caractéristiques écologiques faites sur les sites des relevés. La classification, l'ordination et le test de permutation de l'ACC ont été réalisé avec le logiciel XLSTAT version 2021.1. 


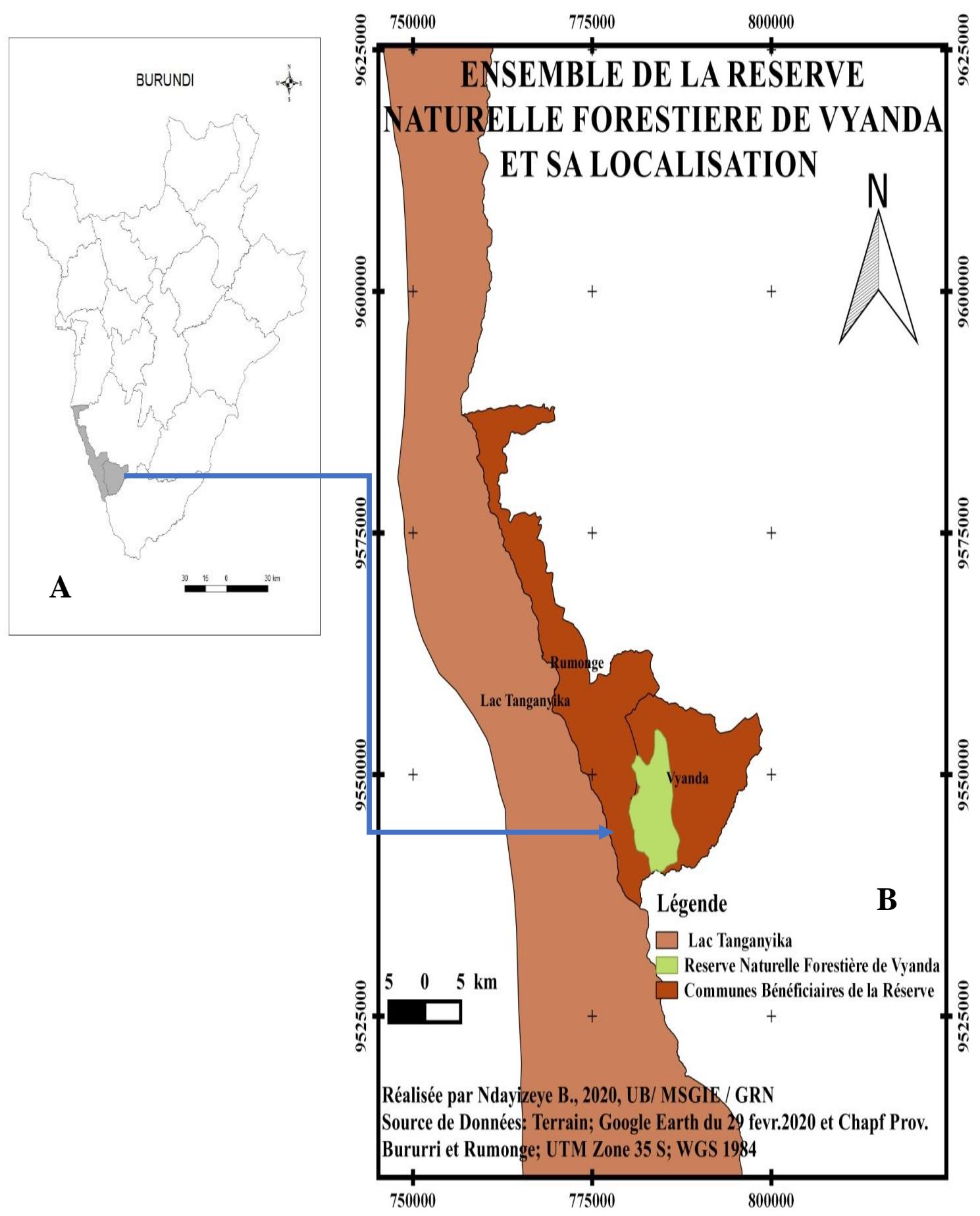

Figure 1 : Localisation de la réserve naturelle forestière de Vyanda (RNFV) : Au sud-Ouest du Burundi (A) et à cheval entre deux entités administratives (B), les communes Vyanda et Rumonge. 
RESULTATS

\section{Composition et richesse spécifique}

L'inventaire floristique de la végétation de la Réserve Naturelle Forestière de Vyanda indique une richesse spécifique de 108 espèces dont 73 dicotylédones, 31 monocotylédones et 4 ptéridophytes; réparties en 81 genres et 41 familles.

Les familles les mieux représentées en espèces et en genres (au moins cinq genres et cinq espèces) sont les Fabaceae, Asteraceae, Rubiaceae, Poaceae ainsi que la famille des Phyllanthaceae. Vingt-six familles (soit plus de 63\%) sont monospecifiques (Tableau 1).

Les genres les plus représentés en espèces sont les genres «Indigofera » avec 7 espèces, "Brachystegia avec 5 espèces et «Aspilia » avec 4 espèces.

\section{Diversité floristique}

Les indices de diversité de Shannon et d'équitabilité de Pielou calculés pour la Réserve Naturelle Forestière de Vyanda sont relativement élevés car supérieurs à la moitié de leur valeur maximale respectivement 3,375 et 0,5 (la valeur maximale l'indice de diversité de Shannon est égale à $\log _{2} S=6,75$, la valeur maximale de l'indice d'équitabilité $=1$ ). Cela est confirmé par l'indice de Simpson (D) faible (Tableau 2).

\section{Forme biologiques, position chronologique et types de diaspores}

Les spectres des formes biologiques des plantes de la réserve naturelle forestière de Vyanda montrent une nette dominance de la forme phanérophytique suivie en ce qui est des proportions par les hémicryptophytes et les chaméphytes. Les géophytes et les thérophytes sont bien représentés avec respectivement $(12,6 \%)$ et $(15,5 \%)$ des espèces recensées. Le spectre brut des types géographiques montre que les espèces de la zone soudanozambéziennes sont fréquentes et le spectre pondéré montre une dominance des espèces paléo tropicales. Ces spectres montrent une bonne représentation des espèces des zones guinéo-congolaises et des espèces afrotropicales. Les spectres brut et pondéré des types de diaspores montrent des proportions élevées pour les sarcochores, puis les sclérochores et les Ballochores. Le reste des types de diaspores n'atteignent pas $15 \%$ pour les proportions pondérées (Figure 2).

\section{Identification des groupements}

Le dendrogramme généré par la classification ascendante hiérarchique sur un tableau de présence/absence formé de 34 relevés et 48 espèces présentes dans au moins 3 relevés permet de mettre en évidence trois groupements végétaux comprenant respectivement 9 relevés, 11 relevés et 13 relevés (Figure 3).

L'indice de similitude est relativement élevé entre tous groupements comparés (Tableau 3). Il est partout supérieur à 50\%. L'indice de similitude est beaucoup plus élevé entre le $2^{\text {ème }}$ et le $3^{\text {ème }}$ groupement qu'entre ces groupements comparés au $1^{\text {er }}$ groupement.

Le nuage de points générés dans le $1^{\text {er }}$ plan factoriel par ordination permet de voir que les espèces se rangent beaucoup plus sur l'axe 1. L'inertie cumulée des deux axes est inférieure à $25 \%$ et les deux axes ne peuvent pas expliquer à eux seuls les groupements végétaux identifiés (Figure 4).

Le test de permutation de l'analyse canonique des correspondances confirme $(\mathrm{P}<$ $0,0001)$ que les données environnementales considérées (classes d'altitude, classes de pente, texture du sol, exposition) sont linéairement liées aux données des espèces. 
Tableau 1 : Nombre d'espèces, de genres et familles inventoriés dans la végétation de la réserve naturelle forestière de Vyanda.

\begin{tabular}{|c|c|c|c|c|c|c|c|}
\hline $\mathbf{N}^{\circ}$ & Famille & $\begin{array}{l}\text { Nombre } \\
\text { Genres }\end{array}$ & $\begin{array}{l}\text { Nombre } \\
\text { espèces }\end{array}$ & $\mathbf{N}^{\circ}$ & Famille (suite) & $\begin{array}{l}\text { Nombre } \\
\text { Genres }\end{array}$ & $\begin{array}{l}\text { Nombre } \\
\text { espèces }\end{array}$ \\
\hline 1 & Fabaceae & 11 & 22 & 22 & Connaraceae & 1 & 1 \\
\hline 2 & Asteraceae & 8 & 11 & 23 & Dennstaedtiaceae & 1 & 1 \\
\hline 3 & Poaceae & 7 & 10 & 24 & Euphorbiaceae & 1 & 1 \\
\hline 4 & Rubiaceae & 8 & 10 & 25 & Gentianaceae & 1 & 1 \\
\hline 5 & Phyllanthaceae & 5 & 6 & 26 & Hypericaceae & 1 & 1 \\
\hline 6 & Acanthaceae & 3 & 3 & 27 & Lauraceae & 1 & 1 \\
\hline 7 & Cyperaceae & 3 & 3 & 28 & Malvaceae & 1 & 1 \\
\hline 8 & Proteaceae & 2 & 3 & 29 & Nephrolepidaceae & 1 & 1 \\
\hline 9 & Combretaceae & 1 & 2 & 30 & Oleandraceae & 1 & 1 \\
\hline 10 & Dioscoreaceae & 1 & 2 & 31 & Orchidaceae & 1 & 1 \\
\hline 11 & Lamiaceae & 1 & 2 & 32 & Oxalidaceae & 1 & 1 \\
\hline 12 & Loganiaceae & 1 & 2 & 33 & Passifloraceae & 1 & 1 \\
\hline 13 & Melastomataceae & 2 & 2 & 34 & Pedaliaceae & 1 & 1 \\
\hline 14 & Myrtaceae & 1 & 2 & 35 & Polygalaceae & 1 & 1 \\
\hline 15 & Smilacaceae & 1 & 2 & 36 & Polygonaceae & 1 & 1 \\
\hline 16 & Anisophylleaceae & 1 & 1 & 37 & Sapindaceae & 1 & 1 \\
\hline 17 & Asparagaceae & 1 & 1 & 38 & Sinopteridaceae & 1 & 1 \\
\hline 18 & Chrysobalanaceae & 1 & 1 & 39 & Tectariaceae & 1 & 1 \\
\hline 19 & Clusiaceae & 1 & 1 & 40 & Vitaceae & 1 & 1 \\
\hline 20 & Colchicaceae & 1 & 1 & 41 & Zingiberaceae & 1 & 1 \\
\hline 21 & Commelinaceae & 1 & 1 & Total & 41 & 81 & 108 \\
\hline
\end{tabular}

Tableau 2 : Valeurs des indices de diversité de Shannon (H), de Simpson (D) et d'Equitabilité de Pielou (E) pour la végétation de la réserve naturelle forestière de Vyanda.

\begin{tabular}{lllll}
\hline Indices de diversité & $\mathbf{H}$ & $\mathbf{D}$ & $\mathbf{E}$ & $\mathbf{S}$ \\
\hline valeur & 4,22 & 0,1 & 0,62 & 108 \\
\hline
\end{tabular}




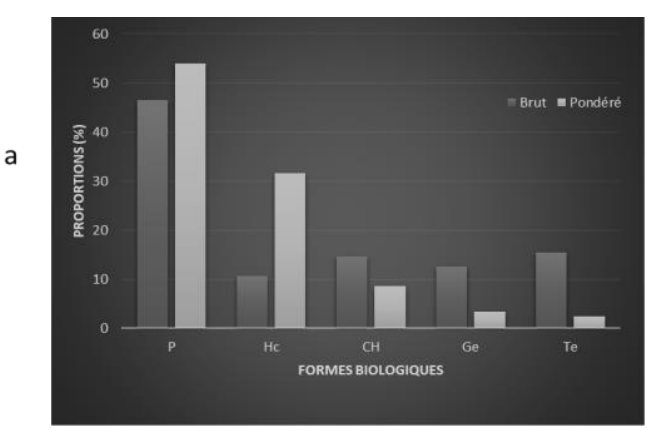

a. P: Phanérophytes, Ch: Chamephytes, Hc: Hémicryptophytes, Ge: Geophytes, Te: Thérophytes

b. SZ: Espèces soudano-zambéziennes, Pan: Espèces pantropicales, Pal: Espèces paléo-tropicales, Afr Trop: Espèces afro-tropicales, Plur Afr: Espèces plurirégionales Africaines, Afr Mal: Espèces afro-malgaches, Mo: Espèces montagnardes, GC: Espèces guinéo-congolaises, Liaison : Espèces de liaison (Mo-SZ, SZ-GC)

c. Sarco: Sarcochores, Scléro: Sclérochores, Ball0: Ballochores, Pogo: Pogonochores, Ptéro: Ptérochores, Baro: Barochores, Desmo: Desmochores b
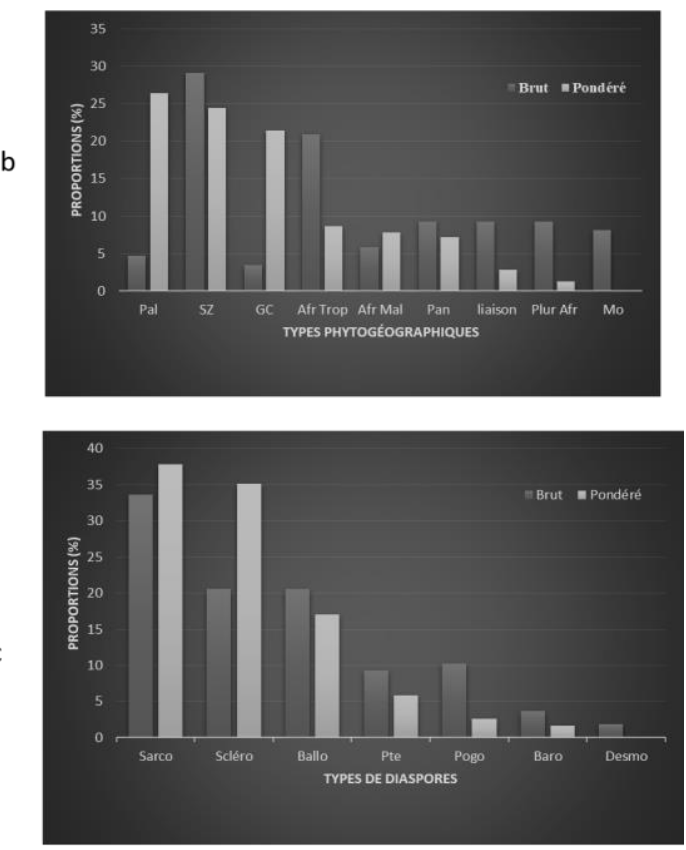

Figure 2 : Spectres bruts et pondérés des formes biologiques (a), des types phytogéographiques (b) et types de diaspores (c).

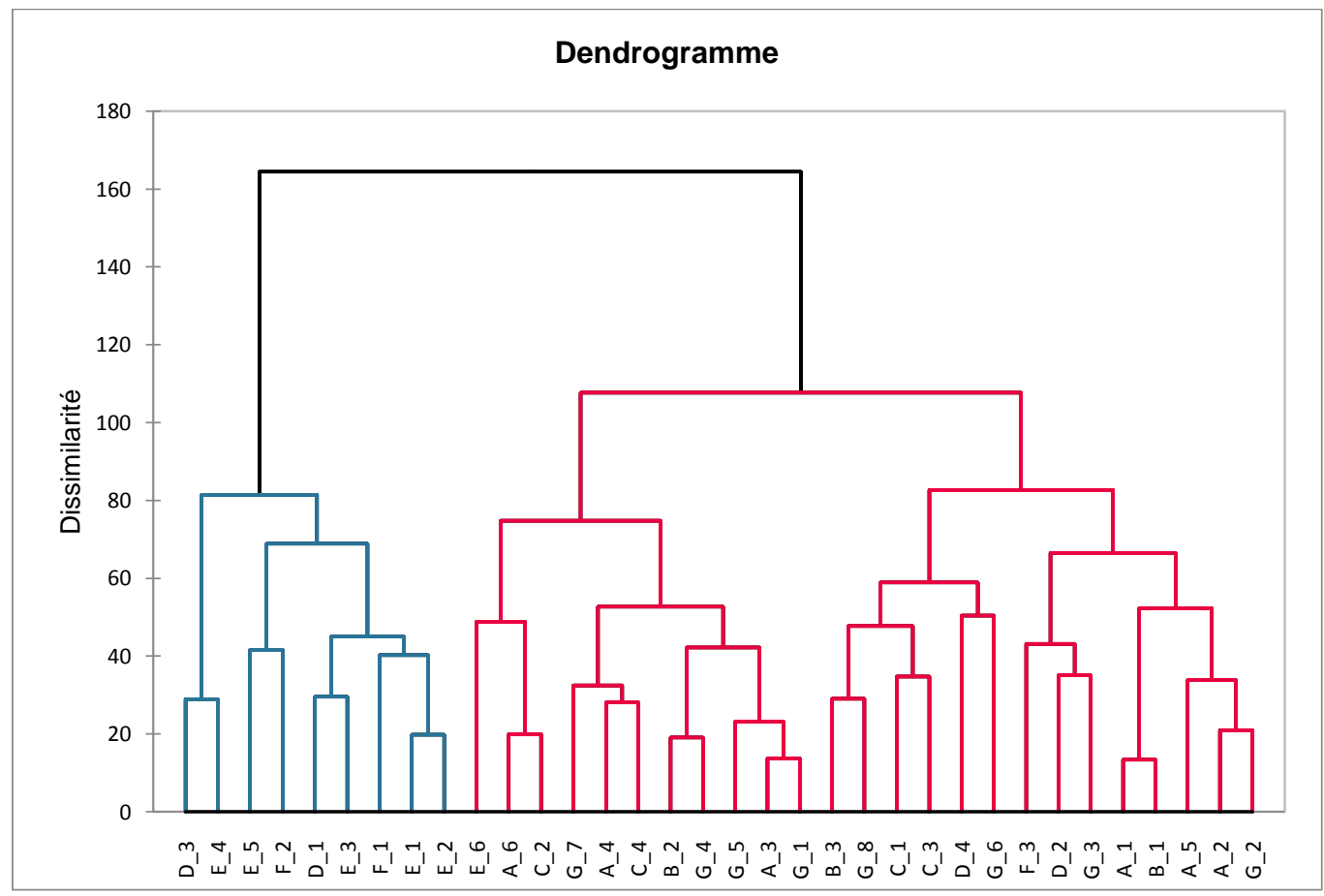

Figure 3 : Classification hiérarchique ascendante des groupements végétaux de la réserve naturelle forestière de Vyanda. Les lettres en majuscules représentent les transects et le numéro associé représente le numéro du relevé sur ce transect. 
Tableau 3 : Similarité (indice de similitude de Sorensen) entre les groupements végétaux identifiés dans la Réserve Naturelle Forestière de Vyanda.

\begin{tabular}{lccc}
\hline & 1er groupement & 2ème groupement & 3ème groupement \\
\hline 1er groupement & $100 \%$ & & \\
2ème groupement & $52,73 \%$ & $100 \%$ & \\
3ème groupement & $52,71 \%$ & $60,47 \%$ & $100 \%$ \\
\hline
\end{tabular}

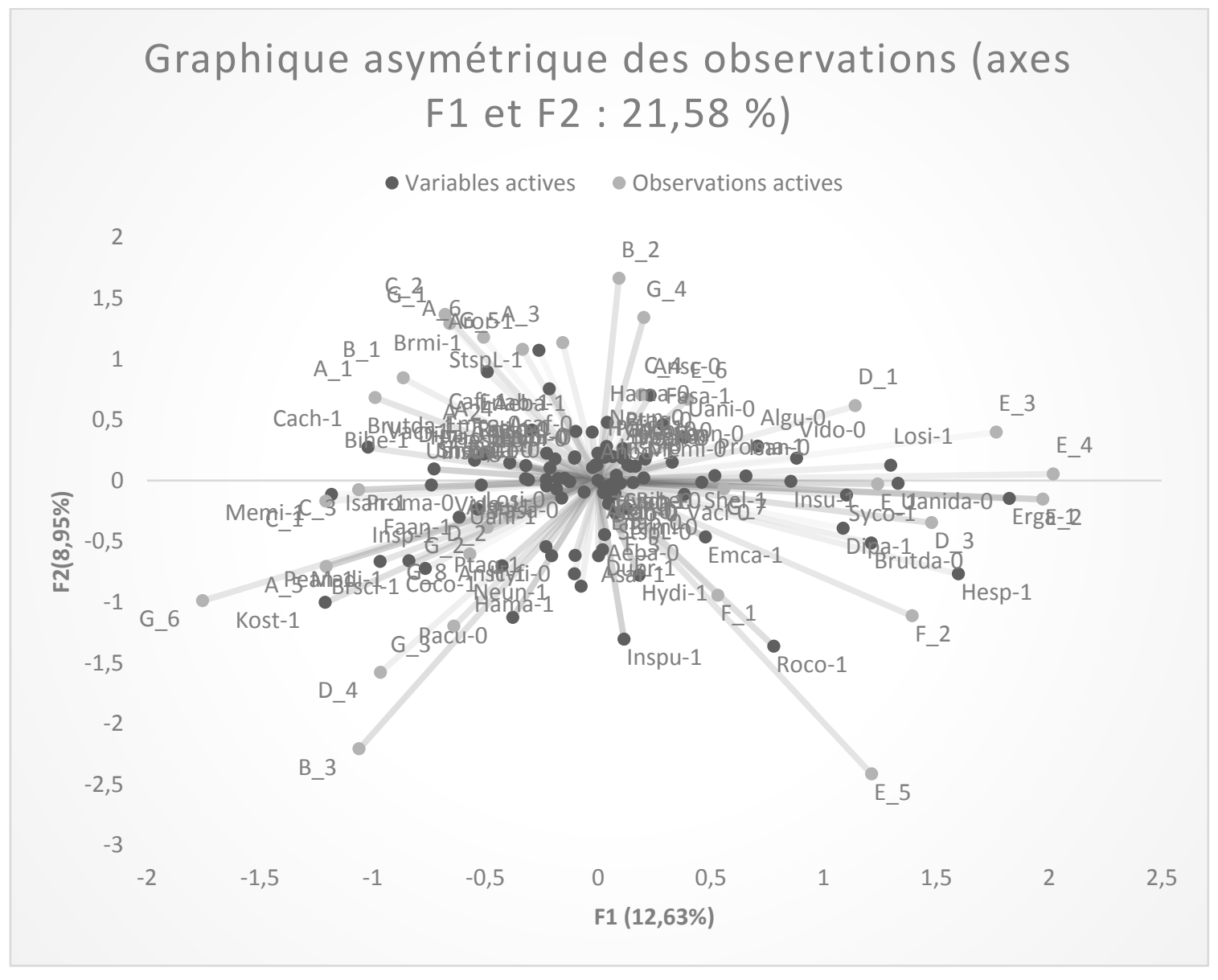

Figure 4 : Premier plan factoriel de l'Analyse Factorielle des Correspondances (AFC) : les lettres en majuscules représentent les transects et le numéro associé représente le numéro du relevé sur ce transect. Les noms des espèces sont en abrégé. 


\section{DISCUSSION}

\section{Composition et diversité floristique}

La composition en terme du nombre des espèces et de genres est presque la même que celles des forêts claires du voisinage qui totalisent 113 espèces réparties en 88 genres et 42 familles (Habonimana et al., 2008). Le nombre d'espèces recensées est faible comparé au nombre recensées dans les autres types d'écosystèmes : 310 espèces recensées au niveau de la forêt péri-gunéenne de Kigwena (Hakizimana, 2012), 522 espèces recensées dans la savane arborée du parc de la Ruvubu (Masharabu et al., 2012), 644 espèces recensées dans la forêt ombrophile de montagne de la Kibira (Nzigidahera, 2000). On peut attribuer cette faiblesse de composition en nombre d'espèces à ce type d'écosystème (forêt claire) qui est une expression des conditions climatiques mais également à l'homogénéité géomorphologique des sites étudiés (colline surplombant la plaine longeant la lac Tanganyika). Cette faiblesse peut également être attribuée à la dégradation anthropique que subit ce milieu. Cela corrobore les résultats de Soumana et al. (2012) et Honvou et al. (2021) quant à l'impact de la dégradation du milieu sur la richesse spécifique de sa végétation. La liste de la flore obtenue peut encore être améliorée en étendant l'inventaire sur des zones non touchées dans le cadre de cette étude.

Les familles des Fabaceae, Asteraceae, Poaceae, Rubiaceae, Phyllanthaceae/ Euphorbiaceae identifiées comme les mieux représentées en espèces, sont celles reconnues les plus représentées dans la flore vasculaire du Burundi et sont aussi citées comme importantes dans d'autres études réalisées au Burundi (Habonimana et al., 2010 ; Masharabu, 2011). La famille des Fabaceae domine les autres familles et cela confirme les résultats de Ndayishimiye et al. (2010) que la famille des Fabaceae est plus diversifiée dans les zones de basses et moyennes altitudes. La prépondérance de la famille des Poaceae met en évidence les effets de la dégradation de la forêt par le feu dont la conséquence est la savanisation (Hakizimana, 2012). De même, les valeurs de l'indice de Shannon $(\mathrm{H})$ et le coefficient d'équitabilité de Pielou sont élevés par rapport à ceux observés pour les forêts claires et cela traduit l'effet des perturbations (Nduwimana et al., 2015).

\section{Spectre des formes biologiques}

Les Phanérophytes sont la forme dominante de la réserve naturelle forestière de Vyanda parce qu'on est en écosystème forêt. L'importance de cette forme est aussi une conséquence des arbres de la famille des Fabaceae (Albizia gummifera, Dalbergia nitidula, Eriosema psoraleoides, Pericopsis angolensis, Brachystegia utilis, Brachystegia microphylla Harms. L'impotance des hémicryptophytes $(31,2 \%)$ représentés par les poaceae (Panicum nervatum, Hyparrhenia diplandra, Hyparrhenia figariana, Hyparrhenia nyssae, Loudetia simplex, Melinis minutiflora, Eragrostis gangetica) traduit une tendance à la savanisation du milieu (Ndayishimiye et al., 2010). La grande représentation des thérophytes $(15,5 \%$ des espèces) est un signe évident des perturbations (Chermat et al., 2012).

Le feu et les coupes menacent en effet les espèces de Brachystegia, un genre qui domine les forêts claires au sud du Burundi, donnant à la réserve naturelle forestière de Vyanda une physionomie distincte conférée par les espèces de Uapaca, plus résistantes à ces menaces.

Les types de diaspores les plus représentés sont les sarcochores $(37,8 \%)$ suivis des sclérochores et des ballochores. L'impotance des sarcochores traduit la présence des arbres fruitiers et de la dispersion assurée par les animaux frugivores. On trouve en effet que dans la réserve naturelle forestière de Vyanda les espèces fruitières telles que Uapaca nitida, Sapium ellipticum, Polygala albida, Fadogia ancylantha, Tapiphyllum kaessneri, Syzygium cordatum, Strychnos spinosa, Dracaena afromontanta, Faurea speciosa, Parinari curatellifolia, Anisophyllea boehmii, Anthocleista schweinfurthii sont bien représentées. La relative importance des sclérochores $(35,1 \%)$ résulte pour la grande part de la place qu'occupe les espèces de la famille des Poaceae dans ce milieu de forêt ouverte avec une importance des espèces comme Imperata cylindrica, Hyparrhenia diplandra, Hyparrhenia figariana et Hyparrhenia nyssae. La bonne représentation 
des ballochores (17\%) vient de la diversité de la famille des Fabaceae.

Les résultats que nous avons trouvés sont en accord avec ceux des autres recherches qui montrent que la zoochorie et autochorie sont les principales voies de dissémination en forêt alors que l'anémochorie constitue une stratégie principale de dissémination pour les plantes des milieux ouverts (Habiyaremye, 1997; Senterre, 2005; Bangirinama, 2010). La même structure où les espèces sarcochores dominent la végétation forestière de colline alors que les espèces sclérochores dominent la végétation savanicole est observée pour le paysage de la Malagarazi (Nduwimana, 2014).

Le spectre pondéré des types phytogéographiques montre une présence marquée des espèces soudano-zambéziennes $(24,4 \%)$ des espèces à distribution paléotropicales $(26,4 \%)$ et les espèces du type guinéo-congolais $(21,4 \%)$. L'importance de l'élément guinéo-congolais est donnée par le genre Uapaca dont la zone de distribution est en principe la limite méridionale de la Région Guinéenne avec une extension de sa distribution dans la zone zambézienne (Devred, 1957).

Cette importance de l'élément soudanozambézien collobore les résultats des autres études qui rattachent notre zone d'étude au centre d'endémisme zambézien, domaine zambézien (Nzigidahera, 1996 ; Habonimana et al., 2008). Le spectre brut des types phytogéographiques montre également que les espèces à large distribution africaine sont bien représentées avec une présence non négligeable des espèces de liaison. La situation de notre zone d'étude au voisinage de la forêt péri-gunéenne de Kigwena et sur différentes altitudes traduirait cette situation.

\section{Identification des groupements végétaux}

La classification ascendante hiérarchique a permis de reconnaitre trois groupements et l'ordination permet de voir que les espèces se rangent sur l'axe 1 avec des espèces arborées (Isoberlinia angolensis, Entada abyssinica, Koschya strigosa, etc) à gauche, les espèces graminéennes à droite (Loudetia simplex, Eragostris gangetica, Hyparrhenia diplandra, etc.), Cela traduit un gradient en ce qui est de la dégradation.
Le fait que les indices de similarité de Sorensen sont partout supérieurs à 50\%, montre que la végétation de la réserve naturelle forestière de Vyanda constitue encore une seule communauté. Bien que la classification ascendante hiérarchique permette de mettre en évidence une tendance à l'individualisation des groupements probablement à la faveur des facteurs externes comme le feu et les coupes sélectives du bois, cela fait preuve que le milieu garde encore sa spécificité.

L'analyse canonique des correspondances montre que l'impact de ces facteurs externes sur les espèces varie en fonction des conditions environnementales comme la pente, la texture du sol, l'altitude et l'exposition au soleil. L'influence des conditions environnementales sur les impacts des facteurs de dégradation de la biodiversité a été mise en évidence par Milà i Canals et al. (2007).

\section{Conclusion}

Cette étude, réalisée dans une végétation qui n'avait pas encore été étudiée, a permis de se rendre compte qu'elle a une diversité relativement élevée et garde encore la spécificité des forêts claires du type miombo malgré la dégradation dont elle fait objet. Cette dernière joue en effet sur la composition floristique (comme la faible dominance du genre Brachystegia) et pourra à terme jouer sur la répartition spatiale. L'établissement des spectres des types biologiques, types géographiques et types de diaspores a mis en évidence la tendance à la savanisation du milieu (prédominance des espèces sclérochores) et un état de dégradation (forte représentation des ptérophytes et des espèces à large distribution) qui appellent à des mesures urgentes visant la restauration et la bonne gestion de ce milieu naturel.

\section{CONFLIT D'INTERETS}

Les auteurs de cet article déclarent qu'il n'y a aucun conflit d'intérêts pour cette publication.

\section{CONTRIBUTIONS DES AUTEURS}

AN et RH ont contribué à toutes les étapes de ce manuscrit (c'est le premier qui l'a rédigé manuscrit et le second l'a relis pour 
améliorer sa qualité). BN et MH ont contribué à la collecte des données et à la détermination des noms des espèces.

\section{REMERCIEMENTS}

Les auteurs expriment leur gratitude à l'endroit de la Direction Générale de l'Office Burundais pour la Protection de l'Environnement (OBPE) pour l'autorisation accordée de mener cette étude dans la réserve naturelle forestière de Vyanda.

\section{REFERENCES}

Abdourhamane H, Morou B, Rabiou H, Mahamane A. 2013. Caractéristiques floristiques, diversité et structure de la végétation ligneuse dans le centre-sud du Niger: cas du complexe des forêts classées du Dan kada Dodo-Dan Gado. Int. J. Biol. Chem. Sci., 7(3) : 1048-1068. DOI:

http://dx.doi.org/10.4314/ijbcs.v7i3.13

Braun-blanquet J. 1932. Plant Sociology: the Study of PlanCommunities. McGraw-Hill Book Co., Inc. : New York and London.

Dansereau P, Lems K. 1957. The grading of dispersal types in plant communities and their ecological significance. Contribution de l'Institut de Botanique de l’Université de Montréal, 71 : 1-52.

Dengler J, Boch S, Filibeck G, Chiarucci A, Dembicz I, Riccardo GR, Henneberg B, Monika JM, Marcenò C, Alireza NA, Polchaninova YN, Kiril VK, Biurrun I. 2016. Assessing plant diversity and composition in grass- lands across spatial scales: the standardised EDGG sampling methodology. EDGG Bulletin, 32: 13-30.

Devred R. 1957. Limite phytogéographique occidento-méridionale de la région guinéenne au Kwango. Bull. Jard. Bot. Etat Bruxelles, 27(3) : 417-431. DOI : https://doi.org/10.2307/3667074

Dray S, Chessel D. 2009. L'ordination des tableaux écologiques. Fiche TD avec le logiciel R: Université Lyon 1 : Lyon.

Gérard M. 2012. Analyse écologique de la répartition de la végétation à partir d'une base de données phytosociologiques : exemple de la végétation méditerranéenne. Dumas 00773444
Gillet F. 2000. La Phytosociologie Synusiale Intégrée. Guide Méthodologique (4ème édition revue et corrigée). Université de Neuchâtel : Neuchâtel.

Grall J, Coic N. 2005. Synthèse des Méthodes d'Evaluation de la Qualité du Benthos en Milieu Côtier. Université de Bretagne Occidentale : Brest.

Gueulou N, Coulibaly B, Ouattara ND, N'guessan AK, Ahoba A, Bakayoko A. 2019. Modes de gestion et efficacité de conservation des reliques de forêts naturelles en zone tropicale sèche : cas du Département de Korhogo (Nord, Côte d'Ivoire). Int. J. Biol. Chem. Sci., 13(7) : 3332-3346.

DOI: https://dx.doi.org/10.4314/ijbcs.v13i7.28

Habiyaremye, MKFX. 1997. Etude Phytosociologique de la Dorsale Orientale du Lac Kivu. Musée royal de l'Afrique centrale : Tervuren.

Habonimana B, Bogaert J, Nzigidahera B, Nahimana G. 2008. Analyse comparée de la composition floristique des forêts claires de Nkayamba et Nyamirambo. Bull. Sc. I.N.E.C.N, 6 : 8-12.

Habonimana B, Bogaert J, Nzigidahera B, Nahimana G. 2010. Les forets claires de Nkayamba et de Nyamirambo de la Réserve Naturelle de Rumonge : Phytogéographie et types biologiques. Bull. Sc. I.N.E.C.N, 8 : 44-50.

Habonimana B, Ndihokubwayo N, Habonayo R, Nzigidahera B, Bogaert J. 2011. Essai de détermination des indicateurs de dégradation forestière : Cas de la forêt ombrophile de la Kibira au Burundi. Colloque international organisé par le SIFEE, $16: 1-14$.

Hakizimana P, Bangirinama F, Habonimana B, Bogaert J. 2011. Analyse comparative de la flore de la forêt dense de Kigwena et de la forêt claire de Rumonge au Burundi. Bull. SC. I.N.E.C.N, 9 : 53-61.

Honvou SHS, Aboh BA, Sewade C, Teka O, Gandonou BC, Oumorou M, Sinsin B. 2021. Diversité floristique, structure et distribution des groupements végétaux des parcours d'accueil des transhumants dans la Basse et Moyenne Vallée de l'Ouémé au Bénin. Int. J. Biol. Chem. 
Sci., 15(1): 81-96. DOI: https://dx.doi.org/10.4314/ijbcs.v15i1.8

Idjigberou ES, Tychon B. 2017. Possibilités d'utilisation de Google Earth ${ }^{\mathrm{TM}}$ pour le suivi du couvert forestier de forets claires en zone soudanienne au Bénin. Ann. UP, Série Sci. Nat. Agron., 7(1) : 54-63

INECN. 2008. Modes de Gouvernance et Catégories d'Aires Protégées Actuelles et Futures au Burundi. INECN: Bujumbura.

Lebrun J. 1947. La végétation de la plaine alluviale au Sud du lac Edouard. Exploration du Parc National Albert. Institut des Parcs Nationaux du Congo Belge : Kinshasa.

Masharabu T. 2011. Flore et végétation du Parc National de la Ruvubu au Burundi : diversité, structure et implications pour la conservation. Thèse de doctorat, ULB, Bruxelles, p. 224.

Masharabu T, Bigendako MJ, Nzigidahera B, Mpawenayo B, Lejoly J, Bangirinama F, Bogaert J. 2012. Vascular flora inventory and plant diversity of the Ruvubu national park, Burundi. Adensonia, 34(1) : 155162. DOI : $10.5252 / \mathrm{a} 2012 \mathrm{n} 1 \mathrm{a} 17$

Meddour R. 2011. La méthode phytosociologique sigmatiste ou braunblanqueto- tüxenienne. Université Mouloud Mammeri: Tizi Ouzou.

Milà i Canals L, Romanya J, Cowell SJ. 2007. Method for assessing impacts on life support functions related to the use of "fertile land" in Life Cycle Assessment. $J$. Clean. Prod., 15(15) : 1426-1440. DOI: 10.1016/j.jclepro.2006.05.005

Morey C, Bashfield RK, Skinner HA. 1983. A comparison of cluster analysis techniques within a sequential validation framework. Multivariate Behav. Res., 18(3): 309-329. DOI:

https://doi.org/10.1207/s15327906mbr18 $03 \_4$

Ndayishimiye J, Sibomana S, Bigendako MJ, Lejoly J, Bogaert J. 2010. Diversité et distribution géographique des légumineuses de la flore du Burundi. Bull. SC. I.N.E.C.N, 8 : $16-21$

Ndayishimiye J, Ndayisenga M, Hakizimana P, Havyarimana F, Bangirinama F,
Masharabu T. 2018. Effets potentiels de l'urbanisation sur l'écosystème de la Réserve Naturelle Forestière de Nkayamba (Commune Rumonge, Province de Rumonge, Burundi). Annales_Facsc_UOB, $4: 107-128$.

Nduwimana A. 2014. Caractérisation du paysage naturel de la Malagarazi (Burundi) et approche de Conservation durable de sa biodiversité. Thèse de doctorat, MNHN/CNRS, Paris, p. 269.

Nduwimana A, Riera B, Bizuru E. 2015. Influence des facteurs écoologiques sur la composition et la densité des unités de végétation du paysage naturel de la Malagarazi (Burundi). Rev. Écol. (Terre Vie), 70(3) : 213-230.

Nzigidahera B. 1996. Paysage Protégé de Gisagara- Plan de Gestion. INECN : Gitega.

Nzigidahera B. 2000. Forêt Claire du Burundi: Conditions Ecologiques, Végétation, Termitières et Champignons Ectomycorrhiziques. INECN : Gitega.

Raunkier C. 1934. The Life Form of Plants and their Bearing on Geography, Collected Papers. Clarendon Press : Oxford.

Reekmans M, Niyongere L. 1983. Lexique Vernaculaire des Plantes Vasculaires $d u$ Burundi. Faculté des Sciences de l'Université du Burundi : Bujumbura.

Roux M. 2006. Algorithmes de classification. Université d'Aix-Marseille : Marseille.

Senterre B. 2005. Recherches méthodologiques pour la typologie de la végétation et la phytogéographie des forêts denses d'Afrique tropicale. Thèse de doctorat, ULB, Bruxelles, p. 441.

Soumana I, Mahamane A, Gandou Z, Ambouta JMK, Saadou M. 2012. Vegetation and plant diversity pattern study of Central Eastern Niger grasslands. Int. J. Biol. Chem. Sci., 6(1): 394-407. DOI: http://dx.doi.org/10.4314/ijbcs.v6i1.34

White F. 1979. The guineo-congolian region and its relationships to other phytochoria. Bull. Jard. Bot. Nat. Belg., 49: 11-55.

White F. 1983. The Vegetation of Africa. A Descriptive Memoir to Accompany the UNESCO/AETFAT/UNSO Vegetation Map of Africa. UNESCO: Paris. 